Laurent Bonavero · Cinzia Casagrande · Stéphane Druel

\title{
On covering and quasi-unsplit families of curves
}

Received September 8, 2005 and in revised form October 18, 2005

\begin{abstract}
Given a covering family $V$ of effective 1-cycles on a complex projective variety $X$, we find conditions allowing one to construct a geometric quotient $q: X \rightarrow Y$, with $q$ regular on the whole of $X$, such that every fiber of $q$ is an equivalence class for the equivalence relation naturally defined by $V$. Among other results, we show that on a normal and $\mathbb{Q}$-factorial projective variety $X$ with canonical singularities and $\operatorname{dim} X \leq 4$, every covering and quasi-unsplit family $V$ of rational curves generates a geometric extremal ray of the Mori cone $\overline{\mathrm{NE}}(X)$ of classes of effective 1-cycles and that the associated Mori contraction yields a geometric quotient for $V$.
\end{abstract}

Keywords. Covering families of curves, extremal curves, quotient

\section{Introduction}

Let $X$ be a normal complex projective variety. Let $V$ be an irreducible and closed subset of Chow $(X)$ such that any element of $V$ is a 1-cycle, and such that for any point $x \in X$, there exists an element of $V$ passing through $x$. We call $V$ a covering family of 1-cycles on $X$.

The framework is Campana's notion of $V$-equivalence, and the construction of the related rational map defined on $X$ : two points $x, x^{\prime}$ are said to be $V$-equivalent if there exist $v_{1}, \ldots, v_{m} \in V$ such that some connected component of $C_{v_{1}} \cup \cdots \cup C_{v_{m}}$ contains $x$ and $x^{\prime}$, where $C_{v} \subset X$ is the curve corresponding to $v \in V$. By Campana's results (see Section 2), there exists a dominant almost holomorphic map $q: X \rightarrow Y, Y$ a normal projective variety, whose general fibers are $V$-equivalence classes. Let $f_{V}:=\operatorname{dim} X-$ $\operatorname{dim} Y$.

A morphism $q^{\prime}: X \rightarrow Y^{\prime}$ onto a normal projective variety $Y^{\prime}$ will be called a geometric quotient for $V$ if every fiber of $q^{\prime}$ is a $V$-equivalence class. If such a quotient exists, then it is clearly unique up to isomorphism. On the other hand, even if $X$ is smooth, a

L. Bonavero, S. Druel: Institut Fourier, UFR de Mathématiques, Université de Grenoble 1, UMR 5582, BP 74, 38402 Saint Martin d'Hères, France;

e-mail: bonavero@ujf-grenoble.fr, druel@ujf-grenoble.fr

C. Casagrande: Dipartimento di Matematica, Università di Pisa, Largo Bruno Pontecorvo 5, 56127 Pisa, Italy; e-mail: casagrande@dm.unipi.it

Mathematics Subject Classification (2000): 14E30, 14J99, 14M99 
geometric quotient for $V$ does not necessarily exist (see Example 22). We refer to [KS02] for a general introduction to this question and related ones.

The main problem is to find sufficient conditions on $X$ and $V$ for the geometric quotient to exist.

Let $\mathcal{N}_{1}(X)_{\mathbb{R}}$ (respectively $\mathcal{N}_{1}(X)_{\mathbb{Q}}$ ) be the vector space of 1 -cycles in $X$ with real (respectively rational) coefficients, modulo numerical equivalence. In $\mathcal{N}_{1}(X)_{\mathbb{R}}$, let $\overline{\mathrm{NE}}(X)$ be the closure of the convex cone generated by classes of effective 1-cycles in $X$.

We say that $V$ is quasi-unsplit if there exists a half-line $R_{V} \subseteq \overline{\mathrm{NE}}(X)$ such that the numerical class of every irreducible component of every cycle in $V$ belongs to $R_{V}$ [CO04, Definition 2.13]. Equivalently, $V$ is quasi-unsplit if all irreducible components of the cycles parametrized by $V$ are numerically proportional.

The first result is the following theorem.

Theorem 1. Let $X$ be a normal and $\mathbb{Q}$-factorial complex projective variety of dimension $n$ and let $V$ be a covering and quasi-unsplit family of 1-cycles on $X$. If $f_{V} \geq n-2$, then there exists a geometric quotient for $V$.

Next, we specialize to rational 1-cycles on $X$, that is, cycles whose irreducible components are rational curves, and look at related questions.

A geometric extremal ray of the Mori cone $\overline{\mathrm{NE}}(X)$ is a half-line $R \subseteq \overline{\mathrm{NE}}(X)$ such that if $\gamma_{1}+\gamma_{2} \in R$ for some $\gamma_{1}, \gamma_{2} \in \overline{\mathrm{NE}}(X)$, then $\gamma_{1}, \gamma_{2} \in R$.

Let $V$ be a covering and quasi-unsplit family of rational 1-cycles on $X$. Is $R_{V}$ a geometric extremal ray of $\overline{\mathrm{NE}}(X)$ ?

Note that this question is natural, since any family of rational 1-cycles such that the general member generates a geometric extremal ray of $\overline{\mathrm{NE}}(X)$ is quasi-unsplit. If $V$ is not assumed to be covering, the preceding statement is not true by looking at a smooth blow-down of a smooth projective variety to a nonprojective one: contracted curves do not define a geometric extremal ray.

Theorem 2. Let $X$ be a normal and $\mathbb{Q}$-factorial complex projective variety of dimension $n$ with canonical singularities. Let $V$ be a covering and quasi-unsplit family of rational 1-cycles on $X$, and let $f_{V}$ be the dimension of a general $V$-equivalence class. If $f_{V} \geq n-3$, then $R_{V}$ is extremal in the sense of Mori theory and the associated contraction yields a geometric quotient for $V$.

We then immediately get the following corollary.

Corollary 1. Let $X$ be a normal and $\mathbb{Q}$-factorial complex projective variety of dimension $\leq 4$ with canonical singularities and let $V$ be a covering and quasi-unsplit family of rational 1-cycles on $X$. Then $R_{V}$ is extremal in the sense of Mori theory and the associated contraction yields a geometric quotient for $V$.

We finally consider the toric case, where we can prove both extremality and existence of a geometric quotient for a quasi-unsplit family of 1-cycles in any dimension. 
Theorem 3. Let $X$ be a toric and $\mathbb{Q}$-factorial complex projective variety, and let $V$ be a covering and quasi-unsplit family of 1-cycles in $X$. Then $R_{V}$ is an extremal ray of $\overline{\mathrm{NE}}(X)$ in the sense of Mori theory and the associated contraction yields an equivariant and equidimensional morphism $q^{\prime}: X \rightarrow Y^{\prime}$ onto a toric and $\mathbb{Q}$-factorial projective variety $Y^{\prime}$ which is a geometric quotient for $V$.

The following is an immediate application of Theorems 2 and 3

Corollary 2. Let $X \subset \mathbb{P}^{N}$ be a normal and $\mathbb{Q}$-factorial variety such that through any point of $X$ there is a line contained in $X$. Assume that either $X$ is toric, or has canonical singularities and $\operatorname{dim} X \leq 4$. Let $V$ be an irreducible family of lines covering $X$. Then there exists a geometric quotient for $V$.

Note that if $X \subset \mathbb{P}^{N}$ has sufficiently small degree then $X$ is covered by lines (see [KNS05] for a precise statement).

Finally, we point out that our results are related to the construction in [BCE+02$]$ of the reduction morphism for a nef line bundle $L \in \operatorname{Pic} X$ (see also [Tsu00]). This is an almost holomorphic rational map $f: X \rightarrow T$, dominant with connected fibers, such that:

○ $L \cdot C=0$ for any curve $C$ contained in a proper fiber $F$ of $f$ with $\operatorname{dim} F=\operatorname{dim} X-$ $\operatorname{dim} Y$

- $L \cdot C>0$ for every irreducible curve $C$ passing through a general point of $X$.

The map $f$ is unique up to birational equivalence of $T$, and the dimension of $T$ is called the nef dimension of $L$.

It is still quite unclear in which circumstances $f$ can be chosen holomorphic (see $\left.\left[\mathrm{BCE}^{+} 02, \S 2.4\right]\right)$; it is always so if the nef dimension of $L$ is at most one. Theorem 1 gives a partial answer for nef line bundles with nef dimension two.

Corollary 3. Let $X$ be a normal and $\mathbb{Q}$-factorial projective variety with $\operatorname{dim} X \geq 3$, and $L \in \operatorname{Pic} X$ be a nef line bundle with nef dimension 2. Assume that $\{\gamma \in \overline{\mathrm{NE}}(X) \mid \gamma \cdot L=0\}$ is a half-line. Then there exists a nef reduction morphism $q: X \rightarrow Y$ onto a normal projective surface $Y$ such that for any curve $C \subset X$ we have $q(C)=\{p t\}$ if and only if $C \cdot L=0$.

\section{Set-up on families of 1-cycles}

Let $X$ be a normal, irreducible, $n$-dimensional complex projective variety. For any curve $C \subset X$, we denote by $[C] \in \mathcal{N}_{1}(X)_{\mathbb{R}}$ its numerical class.

If $R \subset \overline{\mathrm{NE}}(X)$ is a half-line and $D$ is a divisor in $X$, we write $D \cdot R>0, D \cdot R=0$, or $D \cdot R<0$ if respectively $D \cdot \gamma>0, D \cdot \gamma=0$, or $D \cdot \gamma<0$ for a nonzero element $\gamma \in R$.

Let $V$ be a covering family of 1-cycles on $X$. We have a diagram given by the incidence variety $\mathcal{C}$ associated to $V$ :

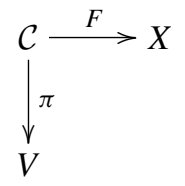

where $\pi$ and $F$ are proper and surjective. Set $C_{v}:=F\left(\pi^{-1}(v)\right)$ for any $v \in V$. 
The relation of $V$-equivalence on $X$ induced by such a family was introduced and studied in [Cam81]; we refer the reader to [Cam04], [Deb01, §5.4], [KMM92] or [Kol96. $\S I V .4]$ for more details. By [Deb01, Theorem 5.9] there exists a closed and irreducible subset of Chow $(X)$ whose normalization $Y$ has the following properties:

(a) if $Z \subset Y \times X$ is the restriction of the universal family,

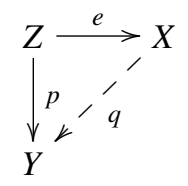

then $e$ is a birational morphism and $q=p \circ e^{-1}$ is almost holomorphic (which means that the exceptional locus of $e$ does not dominate $Y$ );

(b) a general fiber of $q$ is a $V$-equivalence class,

(c) a general fiber of $q$, hence of $p$, is irreducible.

As a consequence of the existence of the map $q$, a general $V$-equivalence class is a closed subset of $X$. We denote by $f_{V}$ its dimension, so that $\operatorname{dim} Y=n-f_{V}$. Moreover, it is well known that any $V$-equivalence class is a countable union of closed subsets of $X$.

Example 1 (see [Kac97, Example 11.1], and references therein). Fix a point $x_{0}$ in $\mathbb{P}^{3}$ and let

$$
P_{0}:=\left\{\Pi \in\left(\mathbb{P}^{3}\right)^{*} \mid x_{0} \in \Pi\right\} \simeq \mathbb{P}^{2}
$$

be the variety of 2-planes in $\mathbb{P}^{3}$ containing $x_{0}$. Consider the variety $X \subset \mathbb{P}^{3} \times P_{0}$ defined as

$$
X:=\left\{(x, \Pi) \in \mathbb{P}^{3} \times P_{0} \mid x \in \Pi\right\} .
$$

Then $X$ is a smooth Fano 4-fold, with Picard number 2 and pseudo-index 2. The two elementary extremal contractions are given by the projections on the two factors.

The morphism $X \rightarrow P_{0}$ is a fibration in $\mathbb{P}^{2}$ : the fiber over a point is the plane corresponding to that point.

Consider the morphism $X \rightarrow \mathbb{P}^{3}$. If $x \neq x_{0}$, the fiber over $x$ is the $\mathbb{P}^{1}$ of planes containing $x$ and $x_{0}$. But the fiber $F_{0}$ over $x_{0}$ is naturally identified with $P_{0}$, hence it is isomorphic to $\mathbb{P}^{2}$. Let $V \rightarrow \mathbb{P}^{3}$ be the blow-up of $x_{0}$ and $\mathcal{C} \rightarrow X$ be the blow-up of $F_{0}$ :

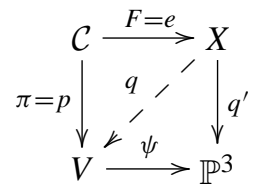

Definition 1. We say that a subset of $X$ is $V$-connected if it is contained in a $V$-equivalence class.

Lemma 1. Let $X$ be a normal projective variety and $V$ be a covering family of 1-cycles on $X$. Consider the diagram (2) above. Then $e\left(p^{-1}(y)\right)$ is $V$-connected for any $y \in Y$. 
Proof. Let $\mathcal{R} \subset X \times X$ be the graph of the equivalence relation defined by $V$; it is a countable union of closed subvarieties since $V$ is proper. The fiber product $Z \times_{Y} Z$ is irreducible and thus $(e \times e)\left(Z \times_{Y} Z\right) \subset \mathcal{R}$ thanks to properties (a) and (b) above. Therefore, for any $x \in e\left(p^{-1}(y)\right)$, the cycle $e\left(p^{-1}(y)\right)$ is contained in the $V$-equivalence class of $x$.

Finally, we will need the following.

Lemma 2. Let $X$ be a normal projective variety and $V$ be a covering and quasi-unsplit family of 1-cycles on $X$. Then there exists a covering and quasi-unsplit family $V^{\prime}$ of 1 -cycles on $X$ such that:

- the general cycle of $V^{\prime}$ is reduced and irreducible;

- for any $v^{\prime} \in V^{\prime}$ there exists $v \in V$ such that $C_{v^{\prime}} \subseteq C_{v}$; in particular $R_{V}=R_{V^{\prime}}$.

Proof. Let $\mathcal{C}$ be the incidence variety associated to $V$ as in (1). It is well known that every irreducible component of $\mathcal{C}$ dominates $V$; let $\mathcal{C}^{\prime \prime}$ be an irreducible component of $\mathcal{C}$ which also dominates $X$. Let $\mathcal{C}^{\prime}$ be the normalization of $\mathcal{C}^{\prime \prime}$ and $\mathcal{C}^{\prime} \rightarrow V^{\prime}$ be the Stein factorization of the composite map $\mathcal{C}^{\prime} \rightarrow \mathcal{C}^{\prime \prime} \rightarrow V$. Since $\mathcal{C}^{\prime} \rightarrow V^{\prime}$ has connected fibers and $\mathcal{C}^{\prime}$ is normal, the general fiber of $\mathcal{C}^{\prime} \rightarrow V^{\prime}$ is irreducible. Moreover, the image in $X$ of every fiber of $\mathcal{C}^{\prime} \rightarrow V^{\prime}$ is contained in a cycle of $V$.

Since $V^{\prime}$ is normal, there is a holomorphic map $V^{\prime} \rightarrow \operatorname{Chow}(X)$. Then after replacing $V^{\prime}$ by its image in $\operatorname{Chow}(X)$ and $\mathcal{C}^{\prime}$ by its image in $\operatorname{Chow}(X) \times X$, we get the desired family.

\section{Properties of the base locus}

Let $V$ be a covering family of 1-cycles on $X$, and recall the diagram (2) associated to $V$. Let $E \subset Z$ be the exceptional locus of $e$, and $B:=e(E) \subset X$. Observe that since $X$ is normal, $\operatorname{dim} B \leq n-2$.

Proposition 1. Let $X$ be a normal and $\mathbb{Q}$-factorial projective variety, and $V$ be a covering and quasi-unsplit family of 1-cycles on X. Consider the associated diagram as in (2). Then:

(i) $e\left(p^{-1}(y)\right)$ is a $V$-equivalence class of dimension $f_{V}$ for every $y \in Y \backslash p(E)$;

(ii) $B$ is the union of all $V$-equivalence classes of dimension greater than $f_{V}$.

Proof. Set $X^{0}:=X \backslash B$ and $Y^{0}:=Y \backslash p(E)=q\left(X^{0}\right)$. Choose a very ample line bundle $H$ on $Y$, and let $U \subset|H|$ be the open subset of prime divisors $D$ such that $D \cap Y^{0} \neq \emptyset$. For any $D$ in $U$, we define $\widehat{D}:=\overline{q^{-1}\left(D \cap Y^{0}\right)}$, which is a prime Weil divisor in $X$. Since $X$ is $\mathbb{Q}$-factorial, some multiple of $\widehat{D}$ defines a line bundle $\widehat{H}$ on $X$. Observe that as $D$ varies in $U$, the divisors $\widehat{D}$ are numerically equivalent in $X$.

Observe also that a general cycle $C_{v}$ of $V$ is contained in a fiber of $q$ disjoint from $\widehat{D}$, so $\widehat{D} \cdot C_{v}=0$. Since $V$ is quasi-unsplit, this gives $\widehat{D} \cdot R_{V}=0$, meaning that for every irreducible component $C$ of every cycle of $V$ we have $\widehat{D} \cdot C=0$. 
Let now $N:=h^{0}(H)$, and let $s_{1}, \ldots, s_{N}$ be general global sections generating $H$. For each $i=1, \ldots, N$, let $D_{i} \in|H|$ be the divisor of zeros of $s_{i}$ and $\widehat{D}_{i}$ in $X$ as defined above.

Let us show that $\widehat{D}_{1} \cap \cdots \cap \widehat{D}_{N}=B$. If $x \notin B$, then $q$ is defined at $x$ and there is some $i_{0} \in\{1, \ldots, N\}$ such that $q(x) \notin D_{i_{0}}$, so $x \notin \widehat{D}_{i_{0}}$. Conversely, let $x \in B$ and fix $i \in\{1, \ldots, N\}$. Then $e^{-1}(x)$ has positive dimension; let $C \subset Z$ be an irreducible curve such that $e(C)=x$. Recall that $Z \subset Y \times X$, so $p(C)$ is a curve in $Y$. Then $D_{i} \cap p(C) \neq \emptyset$ and $p^{-1}\left(D_{i}\right) \cap C \neq \emptyset$. Now observe that $p^{-1}\left(D_{i}\right)$ does not contain any component of $E$, hence $e\left(p^{-1}\left(D_{i}\right)\right)$ is a divisor in $X$ which coincides with $\widehat{D}_{i}$ over $X \backslash B$. Then $\widehat{D}_{i}=e\left(p^{-1}\left(D_{i}\right)\right)$ and $x \in \widehat{D}_{i}$.

Claim. Let $C$ be an irreducible curve in $X$ such that $\widehat{D} \cdot C=0$ for some $D \in U$. Then either $C \subseteq B$, or $C \cap B=\emptyset$ and $q(C)=\{p t\}$.

In fact, assume that $C$ is not contained in $B$. Since $B=\widehat{D}_{1} \cap \cdots \cap \widehat{D}_{N}$, there exists $i \in\{1, \ldots, N\}$ such that $C$ is not contained in $\widehat{D}_{i}$. Then $C \cap \widehat{D}_{i}=\emptyset$, because $C \cdot \widehat{D}_{i}=0$. Thus $C \cap B=\emptyset$.

Moreover if $q(C)$ is a curve, there exists $D_{0} \in U$ such that $D_{0}$ intersects $q(C)$ in a finite number of points. Then $\widehat{D}_{0}$ intersects $C$ without containing it, a contradiction, again because $C \cdot \widehat{D}_{0}=0$.

This shows that $B$ is closed with respect to $V$-equivalence. In fact, let $C$ be an irreducible component of a cycle of $V$ such that $C \cap B \neq \emptyset$. We have $C \cdot \widehat{D}=0$, so the Claim above implies that $C \subseteq B$.

Consider now a $V$-equivalence class $F \subseteq X$. Since $B$ is closed with respect to $V$ equivalence, either $F \cap B=\emptyset$, or $F \subseteq B$.

Assume that $F \cap B=\emptyset$, and choose an irreducible component $C$ of a cycle of $V$ such that $C \subseteq F$. We have $\widehat{D} \cdot C=0$ for any $D \in U$, hence $q(C)$ is a point by the Claim above. By definition of $V$-equivalence, any two points of $F$ can be joined by a chain of components of cycles of $V$, so we have $q(F)=y_{0} \in Y$, and $F \subseteq e\left(p^{-1}\left(y_{0}\right)\right)$. On the other hand, $e\left(p^{-1}(y)\right)$ is $V$-connected by Lemma 1 , so $F=e\left(p^{-1}\left(y_{0}\right)\right)$. Finally, since $F \cap B=\emptyset$, we must have $y_{0} \in Y^{0}$, so $F$ is a proper fiber of $q$ of dimension $f_{V}$.

For any $x \in X$, let $Y_{x}:=p\left(e^{-1}(x)\right)$ be the family of cycles parametrized by $Y$ and passing through $x$, and $\operatorname{Locus}\left(Y_{x}\right):=e\left(p^{-1}\left(Y_{x}\right)\right)$. Observe that for any $y \in Y_{x}$, the subset $e\left(p^{-1}(y)\right)$ contains $x$ and is $V$-connected by Lemma 1 Hence Locus $\left(Y_{x}\right)$ is $V$-connected for any $x \in X$.

Since $Z \subset Y \times X$, we have $\operatorname{dim} Y_{x}=\operatorname{dim} e^{-1}(x)$. Thus $\operatorname{dim} Y_{x}>0$ if and only if $x \in B$, by Zariski's main theorem. If so, $\operatorname{Locus}\left(Y_{x}\right)$ has dimension at least $f_{V}+1$.

Now let $F$ be a $V$-equivalence class contained in $B$, and $x \in F$. Then $\operatorname{Locus}\left(Y_{x}\right)$ has dimension at least $f_{V}+1$ and is contained in $F$, hence $\operatorname{dim} F \geq f_{V}+1$.

Let us remark that in general, if $V$ is not quasi-unsplit, $B$ is not closed with respect to $V$-equivalence.

Example 2. In $\mathbb{P}^{2}$ fix two points $x, y$ and the line $L=\overline{x y}$. Consider $\mathbb{P}^{2} \times \mathbb{P}^{2}$ with the projections $\pi_{1}, \pi_{2}$ on the two factors, and fix three curves $R_{x}, R_{y}, L^{\prime}$ such that: 
$\circ R_{x}$ is a line in $\mathbb{P}^{2} \times x$ and $R_{y}$ is a line in $\mathbb{P}^{2} \times y$;

○ $\pi_{1}\left(R_{x}\right) \cap \pi_{1}\left(R_{y}\right)$ is a point $z \in \mathbb{P}^{2}$;

○ $L^{\prime}:=z \times L$ is the unique line dominating $L$ via $\pi_{2}$ and intersecting both $R_{x}$ and $R_{y}$.

Let $\sigma: W \rightarrow \mathbb{P}^{2} \times \mathbb{P}^{2}$ be the blow-up of $R_{x}$ and $R_{y}$. In $W$, the strict transform of $L^{\prime}$ is a smooth rational curve with normal bundle $\mathcal{O}_{\mathbb{P}^{1}}(-1)^{\oplus 3}$. Let $X$ be the variety obtained by "flipping" this curve. Then $X$ is a smooth toric Fano 4-fold with $\rho_{X}=4$ (this is $Z_{2}$ in Batyrev's list, see [Bat99, Proposition 3.3.5]).

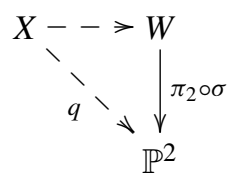

The strict transform of a general line in a fiber of $\pi_{2}$ gives a covering family $V$ of rational curves on $X$. The birational map $X \rightarrow \mathbb{P}^{2} \times \mathbb{P}^{2}$ is an isomorphism over $\mathbb{P}^{2} \times\left(\mathbb{P}^{2} \backslash L\right)$; if $U \subset X$ is the corresponding open subset, then $U$ is closed with respect to $V$-equivalence and every fiber of $q: U \rightarrow \mathbb{P}^{2} \backslash L$ is a $V$-equivalence class isomorphic to $\mathbb{P}^{2}$. Thus $f_{V}=2$.

Let $T_{x}$ and $T_{y}$ be the images in $X$ of the exceptional divisors of $\sigma$ in $W$. These two divisors are $V$-connected, and they cannot be contained in $B$ because $\operatorname{dim} B \leq 2$. Moreover, $P:=T_{x} \cap T_{y}$ is the $\mathbb{P}^{2}$ with normal bundle $\mathcal{O}_{\mathbb{P}^{2}}(-1)^{\oplus 2}$ obtained under the flip. The map $q: X \rightarrow \mathbb{P}^{2}$ cannot be defined over $P$, so $P \cap B \neq \emptyset$. Therefore $B$ cannot be closed with respect to $V$-equivalence.

Observe that the numerical class of $V$ lies in the interior of $\overline{\mathrm{NE}}(X)$, hence the unique morphism, onto a projective variety, which contracts curves in $V$, is $X \rightarrow\{\mathrm{pt}\}$.

Proof of Theorem 1] If $B$ is not empty, Proposition 1 gives $\operatorname{dim} B \geq f_{V}+1 \geq n-1$, which is impossible because $X$ is normal. Hence $B$ is empty and $q: X \rightarrow Y$ is an equidimensional morphism, whose fibers are $V$-equivalence classes.

Proof of Corollary 3 Let $V$ be a covering family of 1-cycles having intersection zero with $L$. Such a family exists because $L$ has nef dimension two and the dimension of $X$ is at least three.

Since $R:=\{\gamma \in \overline{\mathrm{NE}}(X) \mid \gamma \cdot L=0\}$ is an extremal ray of $\overline{\mathrm{NE}}(X)$ and the general 1-cycle of $V$ has numerical class in $R$, the family $V$ is quasi-unsplit and $R_{V}=R$.

Consider the nef reduction $f: X \rightarrow T$ of $L$ and let $F$ be a general fiber. For any irreducible curve $C \subseteq F$ we have $C \cdot L=0$, so $[C] \in R$. Using the Claim in the proof of Proposition 1], we see that $C \cap B=\emptyset$ and $q(C)$ is a point. This is true for all curves in $F$, so $F \cap B=\emptyset$ and $q(F)$ must be point. Then $F$ is contained in a $V$-equivalence class, hence $f_{V} \geq \operatorname{dim} F=n-\operatorname{dim} T \geq n-2$. Now the statement follows from Theorem 1 .

\section{Extremality for covering families of rational 1-cycles}

We now consider extremality properties of $R_{V}$ for a covering and quasi-unsplit family of rational 1-cycles. 
The following well known remark will be of constant use (see [Kol96, Proposition IV.3.13.3], or [ACO04, Corollary 4.2]).

Remark 1. Assume that $V$ is a covering family of rational 1-cycles, and let $Z \subset X$ be $V$-connected. Then every curve contained in $Z$ is numerically equivalent in $X$ to a linear combination with rational coefficients of irreducible components of cycles in $V$. In particular, if $V$ is quasi-unsplit and $C$ is a curve contained in a $V$-connected subset of $X$, then $[C] \in R_{V}$.

A key observation is the following.

Proposition 2. Let $X$ be a normal and $\mathbb{Q}$-factorial projective variety, and $V$ a covering and quasi-unsplit family of rational 1-cycles on $X$. If every connected component of $B$ is $V$-connected, then there exists a nef divisor $\widehat{D}$ on $X$ such that for any curve $C$ in $X$, $\widehat{D} \cdot C=0$ if and only if $[C] \in R_{V}$.

Proof. We use the same notation as in the proof of Proposition 1 . So $H$ is a very ample line bundle on $Y, U \subset|H|$ is the open subset of divisors $D$ that are irreducible and such that $D \nsubseteq p(E)$, and for any $D$ in $U$, we set $\widehat{D}:=\overline{q^{-1}(D \backslash p(E))}$. Recall that $\widehat{D} \cdot R_{V}=0$, and that $B=\widehat{D}_{1} \cap \cdots \cap \widehat{D}_{N}$ for some $D_{1}, \ldots, D_{N} \in U$.

Let us show that $\widehat{D}$ is nef. By contradiction, suppose that there exists an irreducible curve $C$ with $C \cdot \widehat{D}<0$. Then $C$ must be contained in $\widehat{D}_{1}, \ldots, \widehat{D}_{N}$, hence $C \subseteq B$. But $B$ is $V$-connected, so by Remark 1 , $C$ should be numerically proportional to $V$, which is impossible because $\widehat{D} \cdot R_{V}=0$.

Let us finally show that $C \cdot \widehat{\widehat{D}}=0$ if and only if $[C] \in R_{V}$ : actually, if $C \cdot \widehat{D}=0$, the Claim in the proof of Proposition 1 shows that either $C \subset B$ or $C$ is contained in a fiber of $q$, both are $V$-connected, hence $[C] \in R_{V}$ by Remark 1 .

Unfortunately, $B$ is not $V$-connected in general as shown by the following example.

Example 3. Let us go back to Example 1. We have $\mathcal{N}_{F_{0} / X}=\Omega_{\mathbb{P} 2}^{1}(1)$ and $\left(-K_{X}\right)_{\mid F_{0}}=$ $\mathcal{O}_{F_{0}}(2)$. Observe that $V$ is a family of extremal irreducible rational curves of anticanonical degree 2.

If we consider $X \times \mathbb{P}^{1}$ with the same family of curves, we have $\operatorname{dim} Y=4, f_{V}=1$ and $B=F_{0} \times \mathbb{P}^{1}$, which is not $V$-connected.

We finally get the following result: if $B$ has the smallest possible dimension, then it is $V$-connected.

Lemma 3. Let $X$ be a normal and $\mathbb{Q}$-factorial projective variety, and $V$ be a covering and quasi-unsplit family of 1-cycles on $X$. If $\operatorname{dim} B=f_{V}+1$, then every connected component of $B$ is a $V$-equivalence class.

Proof. By Proposition 1. we know that $B$ is the union of all $V$-equivalence classes whose dimension is $f_{V}+1$. Since each of these equivalence classes must contain an irreducible component of $B$, they are in a finite number, and each is contained in a connected component of $B$. 
So if $B_{0}$ is a connected component of $B$, we have $B_{0}=F_{1} \cup \cdots \cup F_{r}$, where each $F_{i}$ is a $V$-equivalence class. We want to show that $r=1$.

Assume by contradiction that $r>1$. Observe that the $F_{i}$ 's are disjoint and $B_{0}$ is connected, hence at least one $F_{i}$ is not a closed subset of $X$; assume it is $F_{1}$.

Then $F_{1}$ is a countable union of closed subsets. Considering the decomposition of $B_{0}$ as a union of irreducible components, we find an irreducible component $T$ of $B_{0}$ such that

$$
T=\bigcup_{m \in \mathbb{N}} K_{m}
$$

where each $K_{m}$ is a nonempty proper closed subset of $T$. Since $T$ is an irreducible complex projective variety, this is impossible.

We reformulate in a single result what we proved so far.

Proposition 3. Let $X$ be a normal and $\mathbb{Q}$-factorial projective variety, and $V$ a covering and quasi-unsplit family of rational 1-cycles on $X$. Then:

(i) either $B=\emptyset$ or $\operatorname{dim} B \geq f_{V}+1$,

(ii) if $B=\emptyset$ or if $\operatorname{dim} B=f_{V}+1$ then there exists a nef divisor $\widehat{D}$ on $X$ such that for any curve $C$ in $X, \widehat{D} \cdot C=0$ if and only if $[C] \in R_{V}$.

\section{Existence of a geometric quotient}

Let $V$ be a covering and quasi-unsplit family of 1-cycles on $X$. Observe that the geometric quotient $q^{\prime}: X \rightarrow Y^{\prime}$ for $V$, provided it exists, has the following property: for any irreducible curve $C$ in $X, q^{\prime}(C)$ is a point if and only if $[C] \in R_{V}$.

Conversely, we show that a morphism with the property above is quite close to being a geometric quotient.

Proposition 4. Let $X$ be a normal and $\mathbb{Q}$-factorial projective variety, and $V$ a covering and quasi-unsplit family of 1-cycles on X. Assume that there exists a morphism with connected fibers $q^{\prime}: X \rightarrow Y^{\prime}$ onto a complete and normal algebraic variety $Y^{\prime}$, such that for any irreducible curve $C$ in $X, q^{\prime}(C)$ is a point if and only if $[C] \in R_{V}$. Then there exists a birational morphism $\psi: Y \rightarrow Y^{\prime}$ that fits into the commutative diagram

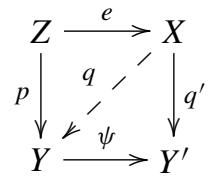

Moreover, if $B^{\prime}:=q^{\prime}(B)$, we have $\left(q^{\prime}\right)^{-1}\left(B^{\prime}\right)=B$, and

$$
B^{\prime}=\left\{y \in Y^{\prime} \mid \operatorname{dim}\left(q^{\prime}\right)^{-1}(y)>f_{V}\right\}=\left\{y \in Y^{\prime} \mid \operatorname{dim} \psi^{-1}(y)>0\right\} .
$$

In particular, every fiber of $q^{\prime}$ over $Y^{\prime} \backslash B^{\prime}$ is a $V$-equivalence class.

Observe that in Example 1, $\psi$ is not an isomorphism. 
Proof. Let $C \subset X$ be an irreducible curve contained in a fiber $F$ of $q^{\prime}$. Then $[C] \in R_{V}$, so the Claim in the proof of Proposition 1 gives that either $C \subseteq B$, or $C \cap B=\emptyset$ and $q(C)$ is a point. Since $F$ is connected, we see that either $F \cap B=\emptyset$, or $F \subseteq B$. This means that $\left(q^{\prime}\right)^{-1}\left(q^{\prime}(B)\right)=B$.

The existence of $\psi$ as in (3) follows easily from the normality of $Y$ and the fact that $q^{\prime}$ contracts all curves in $V$, hence all $V$-equivalence classes. Observe that $\psi$ is surjective with connected fibers.

Let us show that $p$ contracts to a point any fiber of $q^{\prime} \circ e$ over $Y^{\prime} \backslash B^{\prime}$.

Let $F$ be a fiber of $q^{\prime}$ over $Y^{\prime} \backslash B^{\prime}$; then we have $F \subset X \backslash B$. Choose an irreducible curve $C \subseteq e^{-1}(F)$. Then $e(C) \subseteq F$ and $e(C) \cap B=\emptyset$, so $q(e(C))=p(C)$ is a point. Since $e^{-1}(F)$ is connected, we have shown that $p$ contracts $e^{-1}(F)$ to a point. Since $Y$ and $Y^{\prime}$ are normal, this implies that $\psi$ is an isomorphism over $Y^{\prime} \backslash B^{\prime}$.

Finally, let $y \in B^{\prime}$ and let $F^{\prime}=\left(q^{\prime}\right)^{-1}(y)$. Then $F^{\prime} \subseteq B$, so $e$ has positivedimensional fibers on $F^{\prime}$, and $\operatorname{dim} e^{-1}\left(F^{\prime}\right)>\operatorname{dim} F^{\prime} \geq f_{V}$. Since $e^{-1}\left(F^{\prime}\right)=p^{-1}\left(\psi^{-1}(y)\right)$ and $p$ has all fibers of dimension $f_{V}$, we must have $\operatorname{dim} \psi^{-1}(y)>0$.

Proof of Theorem 2 If $B$ is empty, then the statement is clear. Assume that $B$ is not empty. Then Proposition 3 and Lemma 3 show that $\operatorname{dim} B=f_{V}+1=n-2$, every connected component of $B$ is a $V$-equivalence class, and there exists a nef divisor $\widehat{D}$ on $X$ such that for any curve $C$ in $X, \widehat{D} \cdot C=0$ if and only if $[C] \in R_{V}$.

We have to show that $-K_{X} \cdot R_{V}>0$. Let $V^{\prime}$ be the covering family of rational 1cycles on $X$ given by Lemma 2 , and consider a resolution of singularities $f: X^{\prime} \rightarrow X$. The family $V^{\prime}$ determines a covering family $V^{\prime \prime}$ of rational 1-cycles in $X^{\prime}$. If $C_{0} \subset X$ is a general element of the family $V^{\prime}$, then $C^{\prime}:=\overline{f^{-1}\left(C_{0} \backslash \operatorname{Sing}(X)\right)}$ is a general element of $V^{\prime \prime}$, and $C_{0}=f_{*}\left(C^{\prime}\right)$.

Since $C_{0}$ is reduced and irreducible, so is $C^{\prime}$. Moreover $V^{\prime \prime}$ is covering, so $C^{\prime}$ is a free curve in $X^{\prime}$, and it has positive anticanonical degree.

Let $m \in \mathbb{Z}_{>0}$ be such that $m K_{X}$ is Cartier. Since $X$ has canonical singularities, we have

$$
m K_{X^{\prime}}=f^{*}\left(m K_{X}\right)+\sum_{i} a_{i} E_{i}
$$

where $E_{i}$ are exceptional divisors of $f$ and $a_{i} \in \mathbb{Z}_{\geq 0}$. Then

$$
-m K_{X} \cdot C_{0}=-f^{*}\left(m K_{X}\right) \cdot C^{\prime}=-m K_{X^{\prime}} \cdot C^{\prime}+\sum_{i} a_{i} E_{i} \cdot C^{\prime}>0 .
$$

This gives $-K_{X} \cdot R_{V^{\prime}}>0$ and thus $-K_{X} \cdot R_{V}>0$.

Since $X$ has canonical singularities, the cone theorem and the contraction theorem hold for $X$ (see [Deb01, Theorems 7.38 and 7.39]). Moreover, the extremal ray $R_{V}$ lies in the $K_{X}$-negative part of the Mori cone, hence it can be contracted.

Let $q^{\prime}: X \rightarrow Y^{\prime}$ be the extremal contraction; then $Y^{\prime}$ is a normal, projective variety, and it is $\mathbb{Q}$-factorial by [Deb01, Proposition 7.44].

Applying Proposition 4 , we see that all fibers of $q^{\prime}$ over $Y^{\prime} \backslash q^{\prime}(B)$ are $V$-equivalence classes. Since connected components of $B$ are $V$-equivalence classes, they are exactly the fibers of $q^{\prime}$ over $q^{\prime}(B)$, and we have the statement. 


\section{The toric case: proof of Theorem 3}

Step 1: the case with Picard number one.

If $\rho_{X}=1$, the statement is just that $X$ is $V$-connected. This is well known, and can be seen as follows. Consider a divisor $\widehat{D}$ on $X$ constructed as in the proof of Proposition 1 This is an effective divisor which cannot be ample because $\widehat{D} \cdot R_{V}=0$. Since $\rho_{X}=1$, the only possibility is that $Y$ is a point and $\widehat{D}=0$.

Recall the diagram

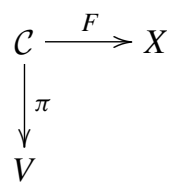

Recall also that if $D \subset X$ is a prime invariant Weil divisor, there is a natural inclusion $i_{D}: \mathcal{N}_{1}(D)_{\mathbb{R}} \hookrightarrow \mathcal{N}_{1}(X)_{\mathbb{R}}$.

Step 2: let $D \subset X$ be a prime invariant Weil divisor such that $D \cdot R_{V}=0$. Then there exists a covering and quasi-unsplit family $V_{D}$ of 1 -cycles in $D$ such that $i_{D}\left(R_{V_{D}}\right)=R_{V}$.

Choose an irreducible component $W$ of $F^{-1}(D)$ which dominates $D$. Set $V_{D}^{\prime}:=$ $\pi(W)$, and let $\mathcal{C}_{D}^{\prime}$ be an irreducible component of $\pi^{-1}\left(V_{D}^{\prime}\right)$ containing $W$. Consider the normalization $\mathcal{C}_{D}$ of $\mathcal{C}_{D}^{\prime}$, and let $\pi_{D}: \mathcal{C}_{D} \rightarrow V_{D}$ be the Stein factorization of the composite map $\mathcal{C}_{D} \rightarrow \mathcal{C}_{D}^{\prime} \rightarrow V_{D}^{\prime}$. Finally, let $F_{D}: \mathcal{C}_{D} \rightarrow X$ be the induced map.

For $v \in V_{D}$, set $G_{v}:=F_{D}\left(\pi_{D}^{-1}(v)\right)$. Then $G_{v} \cap D \neq \emptyset, G_{v}$ is connected, and $G_{v} \cdot D=0$ because $V$ is quasi-unsplit. This implies $G_{v} \subseteq D$, hence $F_{D}\left(\mathcal{C}_{D}\right) \subseteq D$. Moreover, since $W$ dominates $D$, we have $F_{D}\left(\mathcal{C}_{D}\right)=D$.

Since $V_{D}$ is normal, there is a holomorphic map $V_{D} \rightarrow \operatorname{Chow}(D)$. Then after replacing $V_{D}$ by its image in $\operatorname{Chow}(D)$ and $\mathcal{C}_{D}$ by its image in $\operatorname{Chow}(D) \times X$, we get the desired family.

Step 3: if $\rho_{X}>1$, then there exists an invariant prime Weil divisor having intersection zero with $R_{V}$.

In fact, let $q: X \rightarrow Y$ be the rational map associated to $V$. Since $\rho_{X}>1, Y$ is not a point. Let $D$ be a prime divisor in $Y$ intersecting $q\left(X^{0}\right)$ and set $D^{\prime}:=\overline{q^{-1}(D)}$. Since there are curves of the family $V$ disjoint from $D^{\prime}$, we have $D^{\prime} \cdot R_{V}=0$. Moreover, $D^{\prime}$ is linearly equivalent to $\sum_{i} a_{i} D_{i}$, where $a_{i} \in \mathbb{Q}_{>0}$ and $D_{i}$ are invariant prime Weil divisors. Hence the statement.

Step 4: we prove the statement.

Let $\Sigma_{X}$ be the fan of $X$ in $N \cong \mathbb{Z}^{n}$, and let $G_{X}$ be the set of primitive generators of one-dimensional cones in $\Sigma_{X}$. It is well known that $G_{X}$ is in bijection with the set of invariant prime divisors of $X$; for any $x \in G_{X}$, we denote by $D_{x}$ the associated divisor. Recall that for any class $\gamma \in \mathcal{N}_{1}(X)_{\mathbb{Q}}$, we have

$$
\sum_{x \in G_{X}}\left(\gamma \cdot D_{x}\right) x=0 \quad \text { in } N \otimes_{\mathbb{Z}} \mathbb{Q},
$$


and that the assignment $\gamma \mapsto \sum_{x \in G_{X}}\left(\gamma \cdot D_{x}\right) x$ gives a canonical identification of $\mathcal{N}_{1}(X)_{\mathbb{Q}}$ with the $\mathbb{Q}$-vector space of linear relations with rational coefficients among $G_{X}$.

Let $m_{1} x_{1}+\cdots+m_{h} x_{h}=0$ be the relation corresponding to the numerical class of a general cycle of $V$, with $x_{i} \in G_{X}$ and $m_{i}$ nonzero rational numbers for all $i$. Since $V$ is covering and quasi-unsplit, all $m_{i}$ 's must be positive.

The following two statements are equivalent (see [Rei83, Theorem 2.4] and [Cas03, Theorem 2.2]):

(a) there exists a $\mathbb{Q}$-factorial, projective toric variety $Y^{\prime}$ and a flat, equivariant morphism

$q^{\prime}: X \rightarrow Y^{\prime}$ such that for any curve $C$ in $X, q^{\prime}(C)$ is a point if and only if $[C] \in R_{V}$;

(b) for any $\tau \in \Sigma_{X}$ such that $x_{1}, \ldots, x_{h} \notin \tau$, we have

$$
\tau+\left\langle x_{1}, \ldots, \check{x}_{i}, \ldots, x_{h}\right\rangle \in \Sigma_{X} \quad \text { for all } i=1, \ldots, h .
$$

Let us show (b) by induction on the dimension of $X$.

If $\rho_{X}=1$, we have already shown (a) and hence (b) in Step 1 .

Assume that $\rho_{X}>1$. Observe that if $y \in G_{X}$, we have $D_{y} \cdot R_{V}=0$ if and only if $y$ is different from $x_{1}, \ldots, x_{h}$. So by Step 3 , we know that $G_{X} \backslash\left\{x_{1}, \ldots, x_{h}\right\}$ is nonempty.

Clearly, it is enough to check (4) for any maximal $\tau$ in $\Sigma_{X}$ not containing any $x_{i}$. Since $\left\{x_{1}, \ldots, x_{h}\right\} \subsetneq G_{X}$, such a maximal $\tau$ will have positive dimension.

Let $y \in G_{X} \cap \tau$. We have $D_{y} \cdot R_{V}=0$, so by Step 2 there exists a quasi-unsplit, covering family $V_{D_{y}}$ in $D_{y}$ such that $i_{D_{y}}\left(R_{V_{D_{y}}}\right)=R_{V}$.

Set $\bar{N}:=N / \mathbb{Z} \cdot y$ and for any $z \in N$, write $\bar{z}$ for its image in $\bar{N}$. The fan $\Sigma_{D_{y}}$ of $D_{y}$ is given by the projections in $\bar{N} \otimes_{\mathbb{Z}} \mathbb{Q}$ of all cones of $\Sigma_{X}$ containing $y$. The relation corresponding to the numerical class of a general cycle of $V_{D_{y}}$ is $\lambda m_{1} \bar{x}_{1}+\cdots+\lambda m_{h} \bar{x}_{h}$ $=0$ for some $\lambda \in \mathbb{Q}_{>0}$. By induction, we know that (b) holds for $V_{D_{y}}$ in $D_{y}$. In particular, the projection $\bar{\tau}$ of $\tau$ is in $\Sigma_{D_{y}}$, so we have

$$
\bar{\tau}+\left\langle\bar{x}_{1}, \ldots, \overline{\bar{x}}_{i}, \ldots, \bar{x}_{h}\right\rangle \in \Sigma_{D_{y}} \quad \text { for all } i=1, \ldots, h .
$$

This yields (4).

Finally, since $q^{\prime}$ is equidimensional, all fibers must be $V$-equivalence classes and $B=\emptyset$

\section{References}

[ACO04] Andreatta, M., Chierici, E., Occhetta, G.: Generalized Mukai conjecture for special Fano varieties. Central Europ. J. Math. 2, 272-293 (2004) Zbl 1068.14049 MR 2113552

[Bat99] Batyrev, V. V.: On the classification of toric Fano 4-folds. J. Math. Sci. (New York) 94, 1021-1050 (1999) Zbl 0929.14024 MR 1703904

$\left[\mathrm{BCE}^{+}\right.$02] Bauer, T., Campana, F., Eckl, T., Kebekus, S., Peternell, T., Rams, S., Szemberg, T., Wotzlaw, L.: A reduction map for nef line bundles. In: Complex Geometry (Göttingen, 2000), Springer, 27-36 (2002) Zbl 1054.14019 MR 1922095

[Cam81] Campana, F.: Coréduction algébrique d'un espace analytique faiblement Kählérien compact. Invent. Math. 63, 187-223 (1981) Zbl 0436.32024 MR 0610537 
[Cam04] Campana, F.: Orbifolds, special varieties and classification theory: an appendix. Ann. Inst. Fourier (Grenoble) 54, 631-665 (2004) Zbl 1062.14015 MR 2097417

[Cas03] Casagrande, C.: Contractible classes in toric varieties. Math. Z. 243, 99-126 (2003) Zbl pre01922488 MR 1953051

[CO04] Chierici, E., Occhetta, G.: The cone of curves of Fano varieties of coindex four. Preprint math.AG/0401429 (2004)

[Deb01] Debarre, O.: Higher-Dimensional Algebraic Geometry. Universitext, Springer (2001) Zbl 0978.14001 MR 1841091

[Kac97] Kachi, Y.: Extremal contractions from 4-dimensional manifolds to 3-folds. Ann. Scuola Norm. Sup. Pisa Cl. Sci. (4) 24, 63-131 (1997) Zbl 0908.14002 MR 1475773

[KS02] Kachi, Y., Sato, E.: Segre's reflexivity and an inductive characterization of hyperquadrics. Mem. Amer. Math. Soc. 160, no. 763 (2002) Zbl 1039.14016 MR 1938329

[KNS05] Knutsen, A. L., Novelli, C., Sarti, A.: On varieties which are uniruled by lines. Preprint math.AG/0503161 (2005)

[Ko196] Kollár, J.: Rational Curves on Algebraic Varieties. Ergeb. Math. Grenzgeb. 32, Springer (1996) Zbl 0877.14012 MR 1440180

[KMM92] Kollár, J., Miyaoka, Y., Mori, S.: Rational connectedness and boundedness of Fano manifolds. J. Differential Geom. 36, 765-779 (1992) Zbl 0759.14032 MR 1189503

[Rei83] Reid, M.: Decomposition of toric morphisms. In: Arithmetic and Geometry, vol. II: Geometry, Progr. Math. 36, Birkhäuser, 395-418 (1983) Zbl 0571.14020 MR 0717617

[Tsu00] Tsuji, H.: Numerically trivial fibrations. Preprint math.AG/0001023 (2000) 